\title{
La exposición prenatal a andrógenos como factor de reprogramación fetal
}

\author{
Sergio E. Recabarren ${ }^{1 a}$, Teresa Sir-Petermann ${ }^{2}$, Manuel \\ Maliqueo ${ }^{2 b}$, Alejandro Lobos ${ }^{1 c}$, Pedro Rojas-G arcía ${ }^{1 d}$. \\ Prenatal exposure to androgens \\ as a factor of fetal programming
}

Both epidemiological and clinical evidence suggest a relationship between the prenatal environment and the risk of developing diseases during adulthood. The first observations about this relationship showed that prenatal growth retardation or stress conditions during fetal life were associated to cardiovascular, metabolic and other diseases in later life. However, not only those conditions may have lasting effects after birth. Growing evidence suggests that prenatal exposure to steroids (either of fetal or maternal origin) could be another source of prenatal programming with detrimental consequences during adulthood. We have recently demonstrated that pregnant women with polycystic ovary syndrome exhibit elevated androgen levels compared to normal pregnant women, which could provide an androgen excess for both female or male fetuses. We have further tested this hypothesis in an animal model of prenatal androgenization, finding that females born from androgenized mothers have a low birth weight and high insulin resistance, that starts at an early age. On the other hand, males have low testosterone and $\mathrm{LH}$ secretion in response to a $\mathrm{GnRH}$ analogue test compared to control males and alterations in seminal parameters. We therefore propose that our efforts should be directed to modify the hyperandrogenic intrauterine environment to reduce the potential development of reproductive and metabolic diseases during adulthood (Rev Méd Chile 2006; 134: 101-8).

(Key-words: Androgens; Fetal growth retardation; Prenatal exposure delayed effects)

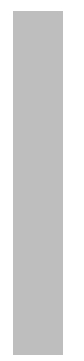
\begin{abstract}
1020232 y \# 1030487.
aiólogo, M.Sc, PhD

bTecnólogo Médico, M.Sc

${ }^{\mathrm{c}}$ Médico Veterinario, M. Sc.

dMédico Veterinario, DVM.
\end{abstract}

Recibido el 28 de octubre, 2004, Aceptado el 3 de marzo, 2005.

${ }^{1}$ Laboratorio de Fisiología y Endocrinología Animal, Facultad de Medicina Veterinaria, Departamento de Ciencias Animales, Universidad de Concepción, Chillán. ${ }^{2}$ Laboratorio de Endocrinología y Metabolismo, Facultad de Medicina Occidente, Universidad de Chile, Santiago. Los trabajos realizados por los autores fueron financiados por los Proyectos Fondecyt \#

Correspondencia a: Dr. Sergio Recabarren. Laboratorio de Fisiología y Endocrinología Animal, Facultad de Medicina Veterinaria, Departamento de Ciencias Animales, Universidad de Concepción, Chillán. 
$\mathrm{S}_{\mathrm{e}}^{\mathrm{e}}$ ha establecido que un órgano o un tejido específico puede ser programado in utero durante un período crítico del desarrollo fetal, con consecuencias adversas en su función en la vida postnatal, lo que se ha definido como reprogramación fetal. Este fenómeno se generaría por efecto de un estímulo o insulto precoz en un periodo sensible del desarrollo fetal, lo que resulta en consecuencias funcionales adversas en la vida adulta. La reprogramación fetal puede comprometer la función metabólica y reproductiva de animales y humanos, actuando en diferentes etapas del desarrollo, modificando el metabolismo glucídico y la función del eje hipotálamo-hipófisis-gónada con consecuencias adversas en la vida post natal. Estudios epidemiológicos han demostrado que existe un aumento en la incidencia del síndrome metabólico y problemas reproductivos en ambos sexos, entre los que cabe destacar la mayor incidencia de niños pequeños para la edad gestacional, el síndrome de ovario poliquístico (SOP) y la pubarquia prematura en la mujer y el compromiso de la función testicular en el hombre, lo que eventualmente podría conducir a una subfertilidad.

La exposición prenatal a andrógenos (EPA), podría actuar como un factor de reprogramación fetal, con efectos adversos sobre la función reproductiva y metabólica, lo cual se ha evaluado fundamentalmente con relación al eje reproductivo de la hembra o de la mujer. En humanos, el modelo clásico de la EPA sería la hiperplasia virilizante congénita. No obstante, un nuevo modelo emerge con la demostración que mujeres SOP presentan elevadas concentraciones de andrógenos durante el embarazo, lo cual podría constituir una fuente anormal de andrógenos para el feto ya sea masculino o femenino. La presente revisión se dirige a discutir los antecedentes de la literatura referente a la reprogramación fetal con especial énfasis en las consecuencias que se observan en el eje reproductivo masculino, con el aporte de nuestras experiencias en humanos y animales.

\section{REPROGRAMACIÓN FETAL}

La reprogramación fetal ha sido definida por Lucas $^{1}$ como el readecuamiento fisiológico por efecto de un estímulo o insulto precoz en un periodo sensible del desarrollo fetal, lo que resulta en consecuencias funcionales adversas a largo plazo. El proceso por el cual ocurre la reprogramación fetal es mediante la inducción, delección o impedimento del desarrollo de ciertas estructuras somáticas como resultado de un estimulo 0 insulto en un periodo crítico ${ }^{1}$.

Es interesante destacar que los mecanismos que conducen a una reprogramación fetal son relativamente limitados y pueden ser comunes para distintas noxas. Estos comprenden cambios en la expresión génica, en la diferenciación de los tejidos y en los procesos homeostáticos como sería la expresión de ciertas enzimas ${ }^{2-5}$.

Una de las primeras observaciones epidemiológicas vinculada a la reprogramación fetal fue la de Barker ${ }^{6}$, quien logró establecer que un cierto patrón de desarrollo intrauterino como es el retardo del crecimiento intrauterino, se relacionaba con una mayor incidencia de patologías específicas de la vida postnatal tales como hipertensión arterial, enfermedad coronaria, insulino resistencia, diabetes tipo 2, dislipidemia, obesidad y alteraciones de tipo reproductivas en los adultos de ambos sexos ${ }^{7,8}$. Se pudo establecer, además, que la asociación entre el retardo del crecimiento intrauterino y estas patologías se presentaba en sujetos adultos de diferente origen étnico, edad y sexo y eran independientes del grado de obesidad y actividad física ${ }^{9,10}$.

Debido a que las hormonas regulan el crecimiento fetal y el desarrollo de tejidos específicos, se propuso que ellas jugarían un papel central en la reprogramación fetal. Hormonas tales como la insulina, los factores de crecimiento insulinosímiles, la tiroxina, los glucocorticoides y los esteroides sexuales (andrógenos y estrógenos), actuarían como señales endocrinas o de maduración, que adaptarían el desarrollo fetal a condiciones intrauterinas adversas, maximizando las condiciones de sobrevida tanto in utero como al nacer, pero a su vez, condicionando un mayor riesgo de ciertas patologías en la vida postnatal ${ }^{9}$.

El papel de las hormonas en la regulación del crecimiento fetal y el desarrollo específico de órganos y tejidos, se ha identificado utilizando diferentes técnicas tales como: ablación de glándulas endocrinas del feto, administración de hormonas al feto o a la madre y knockout de ciertos genes $^{11,12}$. Estos estudios establecen que las hormonas afectan tanto el crecimiento como la 
diferenciación de los tejidos in utero. También establecen que las hormonas pueden actuar directamente (vía genómica) o indirectamente modificando el desarrollo placentario, el metabolismo fetal así como la producción de factores de crecimiento y de otras hormonas por la unidad feto-placentaria ${ }^{13,14}$.

Las hormonas presentes en la circulación fetal pueden tener cuatro orígenes principales. En primer lugar serían las secretadas por las propias glándulas fetales (tales como hormonas tiroideas, pancreáticas, adrenales e hipofisiarias); en segundo lugar, derivarían de los tejidos útero-placentarios (como por ejemplo esteroides, péptidos y glicoproteínas) que son secretados tanto a la circulación umbilical como a la circulación uterina ${ }^{15}$; en tercer lugar, las hormonas lipolíticas tales como esteroides y hormonas tiroídeas que pasarían desde la madre por difusión transplacentaria. La cantidad de hormona transferida por esta vía depende de la gradiente materno-fetal y de la permeabilidad de la barrera placentaria, las que varían según la especie16. Por último, las hormonas presentes en la circulación fetal derivarían de precursores que son metabolizados en el feto o en la placenta.

La concentración de hormonas en la circulación fetal varía normalmente con el desarrollo del mismo y en respuesta a estímulos nutricionales y de otra naturaleza. En condiciones experimentales o patológicas, los cambios hormonales dependerán de la magnitud, duración, naturaleza y momento en que se ejerza el estímulo o noxa, lo que alterará el patrón de desarrollo global o específico de ciertos órganos o tejidos del feto.

El papel de las hormonas, o señales endocrinas en el fenómeno de reprogramación, también implica que compuestos o sustancias con efecto endocrino, los denominados «disruptores endocrinos» presentes en el ambiente, puedan afectar el desarrollo fetal, modificando la concentración de los niveles hormonales y la acción hormonal incluyendo su producción, secreción, transporte y metabolismo.

Inicialmente, el término de sdisruptores endocrinos»se aplicó a compuestos químicos exógenos (xenobióticos) que remedaban o bloqueaban a las hormonas esteroidales interactuando con su receptor $^{17}$. De acuerdo a estudios experimentales, ha surgido la hipótesis que compuestos químicos, con efecto masculino o femenino, podrían actuar como disruptores endocrinos produciendo alteraciones en el desarrollo del sistema reproductivo ${ }^{18,19}$. Además, hay que tener presente que las hormonas endógenas del feto pueden actuar como «disruptores endocrinos» cuando se encuentran en concentraciones suprafisiológicas. Por lo tanto, se considera en la actualidad, que un disruptor endocrino es cualquier hormona que produce un desbalance en el sistema endocrino ${ }^{20}$.

Un ejemplo conocido del efecto negativo de un disruptor hormonal sobre la espermatogénesis en la etapa adulta, la constituyen los estrógenos. Se sabe que la exposición prenatal o neonatal de fetos machos a altas concentraciones de estradiol resulta en una disminución del número de células de Sertoli, deficiencia de la espermatogénesis y aumento de la apoptosis (dependiente de la concentración) en la etapa adulta ${ }^{21}$. El uso de dietiletilbestrol en la prevención del parto prematuro, se encontró claramente asociado a un aumento de cáncer testicular, criptorquidia e hipospadia en los hijos varones de las mujeres tratadas $^{22,23}$. Paralelamente, en el varón se ha observado una disminución en el número de espermatozoides en los últimos 50 años, asociado al aumento del uso y presencia ambiental de numerosos compuestos con actividad estrogéni$\mathrm{ca}^{24}$. Estos resultados demuestran claramente que la exposición a concentraciones suprafisiológicas de estrógenos durante el período prenatal, es crítica e induce una reprogramación permanente del eje hipotalamo-hipófisis-gónada, que se va a traducir en una alteración de la función testicular en el adulto. No obstante, el efecto de concentraciones suprafisiológicas de andrógenos durante la etapa fetal sobre la función del eje reproductivo masculino ha sido menos documentada.

\section{EXPOSICIÓN PRENATAL A ANDRÓGENOS Y FUNCIÓN REPRODUCTIVA}

Estudios clínicos y experimentales han demostrado que la exposición prenatal a andrógenos tiene una serie de consecuencias deletéreas que se expresan en la vida adulta. Estudios en animales, establecen que la exposición prenatal a testosterona, conduce a retardo del crecimiento intrauterino, infertilidad, obesidad, insulino resistencia y a 
cambios de conducta de las crías en su vida postnatal ${ }^{25-32}$.

Además, estudios en hembras ovinas androgenizadas in útero, muestran que la exposición a la testosterona durante el periodo crítico del desarrollo cerebral puede adelantar el inicio del aumento característico de la secreción de LH que conduce a la pubertad en la hembra ovina, fenómeno que, además, se hace independiente del fotoperíodo, lo que es una característica del macho. Adicionalmente, la administración de dihidrotestosterona a la madre permite diferenciar el efecto de los andrógenos aromatizables de los no aromatizables, estableciéndose que la aromatización es necesaria para prevenir el alza preovulatoria de la hormona liberadora de gonadotropinas $(\mathrm{GnRH})$, una característica de la hembra33,34.

En humanos, un modelo clásico de EPA es la hiperplasia virilizante congénita por déficit de 21hidroxilasa, una enzima clave en la esteroidogénesis adrenal. En ella, la excesiva producción de andrógenos por la adrenal del feto produce una autoandrogenización lo que en la vida post natal se evidencia por la presencia de genitales ambiguos y trastomos menstruales en las niñas, virilización progresiva y disminución de la fertilidad en nacidos de ambos sexos y, finalmente, aumento de la conducta agresiva semejante a lo descrito en modelos animales ${ }^{35-37}$. $\mathrm{Si}$ bien estos estudios y nuestras observaciones, sugieren un compromiso de la función reproductiva masculina, ésta ha sido menos estudiada que el impacto que genera la EPA sobre el eje reproductivo de la hembra o de la mujer.

Adicionalmente, estudios en modelos animales (monas y ovejas) establecen que las hembras androgenizadas por una exposición prenatal a andrógenos durante la vida intrauterina, como consecuencia de la administración exógena de testosterona a las madres, presentan cambios neuroendocrinos, gonadales y metabólicos, que en su conjunto asemejan a lo descrito en el síndrome de ovario poliquístico ${ }^{26,38-40}$, lo que sugiere que este síndrome pudiera tener, en parte, un origen prenatal, como consecuencia del ambiente esteroidal anormal. Previamente, desde un punto de vista clínico, los primeros indicios que vincularon un posible papel de una exposición excesiva de andrógenos en la vida fetal con el desarrollo ulterior del SOP en la vida postnatal, derivaron de observaciones en mujeres portadoras de un déficit clásico de la 21-hidroxilasa, que fueron adecuadamente tratadas y que por ende presentaron una normalización de sus andrógenos. Durante su vida adulta, las pacientes desarrollaron rasgos típicos del SOP tales como: anovulación, hipersecreción de $\mathrm{LH}$, resistencia a la insulina y aspecto poliquístico de los ovarios. Lo anterior llevó a proponer que el exceso de andrógenos durante la vida fetal ya sea de origen ovárico o adrenal podría constituir un insulto hormonal que sería necesario para el desarrollo del SOP en la vida postnatal ${ }^{41}$. No obstante, los estudios prospectivos en humanos han sido limitados lo que ha dado origen a la utilización de modelos animales.

Basado en las observaciones clínicas y experimentales queda establecido que la EPA jugaría un papel etiopatogénico en el desarrollo del $\mathrm{SOP}^{26}$. No obstante, lo que ha sido menos explorado es si la madre SOP constituye un modelo clínico de EPA para su hijo(a). En el modelo clínico clásico de EPA como es la hiperplasia virilizante congénita, sería la corteza adrenal hiperandrogénica del propio feto, el origen del exceso prenatal de andrógenos, mientras que en el SOP este origen es más incierto, pudiendo ser la gónada fetal la que se activa generando una autoandrogenización como lo observado en la hiperplasia virilizante congénita $^{41}$, o es la madre SOP la que traspasa un exceso de andrógenos al feto semejante a lo que ocurre en el modelo experimental.

Recientemente, hemos establecido que las embarazadas con SOP presentan niveles elevados de andrógenos pudiendo constituir una fuente de exceso de andrógenos para el feto ${ }^{43}$. No obstante, se desconoce si la gónada fetal de estos niños pudiese ser una fuente de excesiva producción de andrógenos condicionando una autoandrogenización similar a lo descrito para el déficit de 21hidroxilasa. Con este objetivo determinamos la concentración de esteroides sexuales en sangre de cordón en hijos/as de madres SOP y controles sanos pareadas por edad, índice de masa corporal y edad gestacional. Contrariamente a lo esperado, pudimos establecer que la concentración de testosterona total era significativamente menor en los hijos varones de madres SOP que en los hijos varones de madres controles lo que hacia poco probable que el testículo fetal de estos niños fuese una fuente de excesiva producción de andrógenos 
para condicionar una autoandrogenización. Por el contrario, los datos más bien sugieren una disfunción de la gónada masculina. A diferencia de los varones, las niñas nacidas de madres con SOP presentaron en comparación a las niñas nacidas de madres controles, concentraciones similares de andrógenos pero concentraciones significativamente más altas de estrógenos (observaciones no publicadas). Con el fin de profundizar en el estudio de la función gonadal de estos niños, estamos evaluando la función del eje reproductivo mediante un test de análogo de GnRH durante el segundo y tercer mes de vida (ventana de activación gonadal postnatal) en hijos e hijas de mujeres con SOP en comparación a hijos e hijas nacidos de mujeres normales. Hemos observado que tanto los hijos como las hijas de mujeres con SOP presentan alteraciones de la esteroidogénesis gonadal lo que se evidencia en los niños por una menor secreción de testosterona al estímulo con análogo de $\mathrm{GnRH}$ y en las niñas por una excesiva producción de estrógenos post-estímulo, lo que es concordante con los resultados obtenidos en sangre de cordón (observaciones no publicadas).

Estas observaciones sugieren que el fenotipo masculino o femenino del SOP podría iniciarse precozmente en ambos sexos y es altamente probable que la androgenización interactúe con alteraciones genéticas pre-existentes. Por lo tanto, en este sentido siguen siendo válidos los modelos experimentales en animales androgenizados in utero con el fin de despejar la incógnita de la real importancia de la androgenización prenatal independiente de un cluster genético.

Teniendo presente estas restricciones, hemos complementado nuestros estudios con un modelo animal de androgenización fetal consistente en la administración de testosterona propionato a ovejas madres entre los días 30 y 90 de preñez (duración de la preñez $=147$ días). Los resultados de esos estudios mostraron que los machos ovinos nacidos de madres androgenizadas con el esquema señalado anteriormente, se caracterizaron por secretar menos testosterona en respuesta al test de análogo de GnRH (test de luprón). Este fenómeno, que se inicia tempranamente en machos de cinco semanas de edad (etapa infantil), se consolida en machos postpuberales de 30 semanas de edad, acompañado de una menor secreción de LH a esa edad (observaciones no publicadas). Por otro lado, resultados preliminares sugieren un retardo en el desarrollo sexual caracterizado por retraso en el inicio de la función gametógenica, un menor recuento espermático, y un menor volumen de semen que en corderos controles lo que concuerda con una probable disfunción gonadal y reafirma los resultados encontrados en niños nacidos de madres con SOP. (observaciones no publicadas).

\section{EXPOSICIÓN PRENATAL A ANDRÓGENOS CAMBIOS METABÓLICOS Y DEL PESO CORPORAL}

Tanto en el modelo humano como en el modelo animal se ha observado una relación entre la exposición prenatal a andrógenos y el retardo del crecimiento intrauterino que por lo general, dará origen a un recién nacido pequeño para la edad gestacional ${ }^{31,46}$. Ambas condiciones producirían una serie de cambios del eje reproductivo y de la homeostasis glucídica del feto los cuales se harían evidentes en su vida postnatal ${ }^{44}$. Se ha planteado que los niños con PEG tienen mayor riesgo de desarrollar síndrome metabólico en la vida adulta y se ha sugerido además, una relación entre el bajo peso de nacimiento y el desarrollo posterior de SOP. A este respecto, hemos podido establecer que el antecedente de PEG es mayor $(18,4 \%)$ en mujeres con SOP que en mujeres cíclicas normales $(7,5 \%)^{45}$. Por otro lado, que la prevalencia de PEG es significativamente mayor en los niños nacidos de madres con SOP $(14,5 \%)$ en comparación a la de madres controles pareadas al inicio del embarazo por edad e índice de masa corporal $(2,0 \%)^{46}$, todo lo cual sugiere una relación entre PEG y SOP como ha sido previamente propuesto ${ }^{47}$. Recientemente, se ha descrito que las hembras nacidas de ovejas androgenizadas tienen un menor peso al nacimiento y un crecimiento compensatorio a los 2-4 meses de vida postnatal ${ }^{31,32}$, lo que no se ha observado en los machos nacidos de hembras androgenizadas. Además las hembras androgenizadas presentan desde temprana edad (5 semanas), hasta el inicio de los ciclos reproductivos (30 semanas de edad), una marcada resistencia a la insulina en comparación con hembras controles ${ }^{31}$. Estos estudios sugieren que, tanto el crecimiento intrauterino como el crecimiento compensatorio durante la vida postnatal pueden ser programados 
por una exposición prenatal a esteroides sexuales. La similitud que presenta la EPA y el retardo del crecimiento intrauterino respecto a las consecuencias a largo plazo, plantea que ambas condiciones podrían tener mecanismos comunes de reprogramación o que en parte las consecuencias de la EPA sean mediadas por un retardo del crecimiento intrauterino ${ }^{32}$. Algunos de los posibles mecanismos por los cuales la EPA podría conducir a un retardo del crecimiento intrauterino serían, en primer lugar, que los andrógenos en exceso puedan aromatizarse a estrógenos, lo que sería particularmente importante en el varón o en el macho. Estudios en humanos han demostrado que la exposición fetal a compuestos estrogénicos se traduce en un retardo del crecimiento intrauterino ${ }^{48-50}$. Un ejemplo clásico es el de los recién nacidos de madres expuestas a dietilbestrol ${ }^{51}$. Un segundo mecanismo es que los andrógenos modifiquen la sensibilidad a la insulina y la acción metabólica de la insulina lo cual ha sido asociado a un retardo del crecimiento intrauterino tanto en humanos como animales ${ }^{52,53}$. Un tercer mecanismo por el cual los andrógenos podrían conducir a un retardo del crecimiento intrauterino sería generando cambios del sistema de factores de crecimiento símil insulina $(\mathrm{IGF})^{54}$ ya sea en la concentración total de IGF-I o de sus proteínas transportadoras con lo cual disminuiría su bioactivi-

\section{REFERENCIAS}

1. LuCAS A. Programming by early nutrition in man. En: Block G.R. and Whelan J, ed. The Childhood Environment and Adult Disease. Editorial John Whiley \& Sons, 1991; 38-55.

2. Desai M, Byrne CD, Meeran K, Martenz ND, Bloom SR, Hales CN. Programming of hepatic insulinsensitive enzymes in offspring of rat dams fed a protein-restricted diet. Am J Physiol 1997; 272: G1083-G1090.

3. Peterside IE, Selak MA, Simmons RA. Impaired oxidative phosphorylation in hepatic mitochondria growth-retarded rats. Am J Physiol Endocrinol Metab 2003; 285: E1258-E1266.

4. Lane RH, Keluey DE, Gruetzmacher EM, Devaskar SU. Uteroplacental insufficiency alters hepatic fatty acid-metabolizing enzymes in juvenile and adult rats. Am J Physiol Regul Integr Comp Physiol 2001; 280: R183-R190. dad. A esto habría que agregar que el sistema IGF está estrechamente relacionado al desarrollo fetal en diferentes especies incluyendo la humana ${ }^{55}$. Finalmente un cuarto mecanismo más hipotético que los anteriores es que los andrógenos actúen como un factor de estrés produciendo una activación del eje adrenal del feto o de la madre y un aumento de glucocorticoides. Estudios en humanos y animales han establecido una asociación entre niveles elevados de cortisol durante la vida fetal y el bajo peso al nacer ${ }^{56-58}$.

En síntesis: La EPA puede actuar como un factor de reprogramación fetal per se 0 a través del un retardo del crecimiento intrauterino el cual, desde un punto de vista clínico, podría servir como un marcador temprano de patologías postnatales, considerando que muchos de los efectos de la reprogramación fetal no se hacen evidentes sino hasta la vida adulta. La EPA, en humanos podría ser un factor de reprogramación fetal frecuente dada la alta prevalencia de los estados hiperandrogénicos como el SOP, la hiperplasia virilizante congénita y el uso de sustancias anabólicas.

Nuestros esfuerzos deben ir encaminados a modificar el ambiente intrauterino hiperandrogénico con el fin de reducir el desarrollo de patologías postnatales de la esfera reproductiva y metabólica.

5. Simmons RA, Flozak AS, Ogata ES. The effect of insulin and insulin-like growth factor-I on glucose transport in normal and small for gestational age fetal rats. Endocrinology 1993; 133: 1361-8.

6. BARKER DJ. Mothers, Babies and Disease in Later Life. London Editorial BMJ Publishing, 1994.

7. De Bruin JP, Dorland M, Bruinse HW, Spliet W, NikKels PG, Te Veide ER. Fetal growth retardation as a cause of impaired ovarian development. Early Hum Dev 1988; 51: 39-46.

8. Ghirri P, Ciu山 C, Vuerich M, Cuttano A, Faraon M, GUERRIN L ET AL. Incidence at birth and natural history of cryptorchidism: a study of 10,730 consecutive male infants. J Endocrinol Invest 2002; 25: 709-15.

9. Barker DJ, Clark P. Fetal undernutrition and disease in later life. Rev Reprod 1997; 2: 105-12.

10. RHIND SM, RAE MT, BRooks AN. Effects of nutrition and environmental factors on the fetal programming of the reproductive axis. Reproduction 2001; 122: 205-14. 
11. FowDEN AL Endocrine regulation of fetal growth. Repr Fert Develop 1995; 7: 351-63.

12. EfSTRATIADis A. Genetics of mouse growth. Int J Dev Biol 1998; 42: 955-76.

13. Fowden AL, ForheAd AJ. The role of hormones in intrauterine development. En Lung Biology in Health and Disease, vol 151. Editorial DJP Barker. New York: Marcel Dekker, 2001; 199-228.

14. FowDEN AL The insulin-like growth factors and feto-placental growth. Placenta 2003; 24: 803-12.

15. Chams JRG, Sloboda D, Matthews SC, Holoway A, Alfraidy N, Howe D et al. The fetal placental hypothalamic-pituitary-adrenal axis, parturition and postnatal health. Mol Cell Endocrinol 2001; 185: 135-44.

16. Sibley C, Giazier J, D'Souza S. Placental transporter activity and expression in relation to fetal growth. Expl Physiol 1997; 82: 389-402.

17. McLachian JA. Environmental signaling: what embryos and evolution teach us about endocrine disrupting chemicals. Endocr Rev 2001; 22: 31941.

18. Mori C. Possible effects of endocrine disruptors on male reproductive function. Kaibogaku Zasshi 2001; 76: 361-8.

19. Anderson LM, Diwan BA, Fear NT, Roman E. Critical windows of exposure for children's health: cancer in human epidemiological studies and neoplasms in experimental animal models. Environ. Health Perspect 2000; 108: Suppl. 3: 573-594.

20. FISHER JS. Environmental anti-androgens and male reproductive health: focus on phthalates and testicular dysgenesis syndrome. Reproduction 2004; 127: 305-15.

21. Atanassova N, Mckinnell C, Walker M, Turner KJ, Fisher JS, Morley M et al. Permanent effects of neonatal estrogen exposure in rats on reproductive hormone levels, Sertoli cell number, and the efficiency of spermatogenesis in adulthood. Endocrinology 1999; 140: 5364-73.

22. OlIVER RT. Germ cell cancer of the testis. Curr Opin Oncol 1998; 10: 266-72.

23. Sharpe RM, MckinNell C, Kivin C, Fisher JS. Proliferation and functional maturation of Sertoli cell, and their relevance to disorders of testis function in adulthood. Reproduction 2003; 125: 769-84.

24. SHARPE RM, SKaKKeBAEK NE. Are oestrogens involved in falling sperm counts and disorders of the male reproductive tract? Lancet 1993; 341: 1392-5.
25. Ciarke IJ, Scaramuzzi RJ, Short RV. Ovulation in prenatal androgenized ewes. J Endocrinol 1977; 73: 385-9.

26. Аввотt DH, Dumesic DA, Eisner JR, Colman RJ, KEMNITZ JW. Insights into the development of polycystic ovary syndrome (PCOS) from studies of prenatally androgenized female rhesus monkeys. Trends Endocrinol Metab 1998; 9: 62-7.

27. OzAnNe SE. Metabolic programming in animals. $\mathrm{Br}$ Med Bull 2001; 60: 143-52.

28. Wolf CJ, Hotchkiss A, Ostby JS, Leblanc GA, Gray Jr LE. Effects of prenatal testosterone propionate on the sexual development of male and female rats: a dose-response study. Toxicol Sci 2002; 65: 71-86.

29. Birch RA, Padmanabhan V, Foster DL, Robinson JE. Prenatal programming of reproductive neuroendocrine function: fetal androgen exposure produces progressive disruption of reproductive cycles in sheep. Endocrinology 2003; 144: 1426-34.

30. Rosser C, Herkimer C, Sarma HN, Recabarren SE, Dunaif A, Padmanabhan V. Fetal programming: prenatal exposure to excess testosterone programs hyperinsulinemia. Biol Reprod 2003; 68 (Suppl 1):293 (Abstract 440).

31. Recabarren SE, Padmanabhan V, Codner E, Lobos A, Durán C, Vidal M ET al. Postnatal developmental consequences of altered insulin sensitivity in female sheep treated prenatally with testosterone. Am J Physiol Endocrinol Metab 2005; 289: 801-6.

32. ManikKam M, Crespi EJ, Doop DD, Herkimer C, LeE JS, Yu S, BRown MB ET AL. Fetal programming: Prenatal testosterone excess leads to fetal growth retardation and postnatal catch-up growth in sheep. Endocrinology 2004; 145: 790-6.

33. Masek KS, Wood RI, Foster DL Prenatal dihydrotestosterone differentially masculinizes tonic and surge modes of luteinizing hormone secretion in sheep. Endocrinology 1999; 140: 3459-66.

34. Sharma TP, Herkimer C, West C, Ye W, Birch R, RoBinson JE ET AL. Fetal programming: prenatal androgen disrupts positive feedback actions of estradiol but does not affect timing of puberty in female sheep. Biol Reprod 2002; 66: 924-33.

35. NEW MI. Factors determining final height in congenital adrenal hyperplasia. J Pediatr Endocrinol Metab 2001; 14(Suppl 2): 933-7.

36. New MI, Carlson A, Obeid J, Marshall I, Cabrera MS, GoSeCO A ET AL. Prenatal diagnosis for congenital adrenal hyperplasia in 532 pregnancies. J Clin Endocrinol Metab 2001; 86: 5651-7. 
37. Berendaum SA, Duck SC, Bryk K. Behavioral effects of prenatal versus postnatal androgen excess in children with 21-hydroxylase-deficient congenital adrenal hyperplasia. J Clin Endocrinol Metab 2000; 85: 727-33.

38. EIsNer JR, Dumesic DA, Kemnitz JW, Авbott DA. Timing of prenatal androgen excess determines differential impairment in insulin secretion and action in adult female rhesus monkeys. J Clin Endocrinol Metab 2000; 85: 1206-10.

39. Eisner JR, Barnett BA, Dumesic DA, Aвbott DH. Ovarian hyperandrogenism in adult female rhesus monkeys exposed to prenatal androgen excess. Fertil Steril 2002; 77: 167-72.

40. Recabarren SE, Sir-Petermann T, Lobos A, Codner E, Rojas-Garcia PP, Reyes V. Response to the gonadotropin releasing hormone agonist leuprolide in immature female sheep androgenized in utero. Biol Res 2005; 38: 235-44.

41. Barnes RB, Rosenfield RL, Ehrmann DA, Cara JF, CUtTLeR L, LevitsKy LL et al. Ovarian hyperandrogenism as a result of congenital adrenal virilizing disorders: evidence for perinatal masculinization of neuroendocrine function in women. J Clin Endocrinol Metab 1994; 79: 1328-33.

42. Аввотт DH, Dumesic DA, FranKs S. Developmental origin of polycystic ovary syndrome - a hypothesis. J Endocrinol 2002; 174: 1-5.

43. Sir-Petermann T, Maliqueo M, Angel B, Lara HE, Perez-Bravo F, Recabarren SE. Maternal serum androgens in pregnant women with polycystic ovary syndrome: possible implications in prenatal androgenization. Hum Reprod 2002; 10: 2573-9.

44. Hofman PL, Cutfield WS, Robinson EM, Bergman RN, Menon RK, SperLing MA, Gluckman PD. Insulin resistance in short children with intrauterine growth retardation. J Clin Endocrinol Metab 1997; 82: 402-6.

45. Benitez R, Sir-Petermann T, Palomino A, Angel B, Malquego M, Pérez-Bravo F et al. Prevalencia familiar de patologías metabólicas en pacientes con síndrome de ovario poliquístico. Rev Méd Chile 2001; 129: 707-12.

46. Sir-Petermann T, Hitschfeld C, Maliqueo M, Codner E, Echiburu B, Gazitua R et al. Birth weight in offspring of PCOS mothers. Human Reprod 2005; 20: 2122-6.
47. IbáÑez L, Potau N, Francois I, de Zegler F. Precocious pubarche, hyperinsulinism and ovarian hyperandrogenism in girls: relation to reduced fetal growth. J Clin Endocrinol Metab 1998; 83: 3558-62.

48. Munger R, Isacson P, Hu S, Burns T, Hanson J, LYNCH CF ET AL. Intrauterine growth retardation in Iowa communities with herbicide-contaminated drinking water supplies. Environ Health Perspect 1997; 105: 308-14.

49. Sharara FI, Seifer DB, Flaws JA. Environmental toxicants and female reproduction. Fertil Steril 1998; 70: 613-22.

50. Giusti RM, Iwamoto K, Нatch EE. Diethylstilbestrol revisited: a review of the long-term health effects. Ann Intern Med 1995; 22: 778-88.

51. Lunn S, Lueberman E, Schoenbaum SC, Monson RR, Stubblefield PG, Ryan KJ. Adverse outcomes of pregnancy in women exposed to diethylstilbestrol in utero. J Reprod Med 1988; 33: 3-7.

52. Boloker J, Gertz SJ, Simmons RA. Gestational diabetes leads to the development of diabetes in adulthood in the rat. Diabetes 2002; 51: 1499-506.

53. Holt RI. Fetal programming of the growth hormone-insulin-like growth factor axis. Trends Endocrinol Metab 2002; 13: 392-7.

54. Briard N, Dadoun F, Pommier G, Sauze N, Lebouc Y, OLVER C ET AL. IGF-I/IGFBPs system response to endotoxin challenge in sheep. J Endocrinol 2000; 164: 361-9.

55. KJos SL, Buchanan TA. Gestational diabetes mellitus. N Engl J Med 1999; 341: 1749-56.

56. SECKL JR. Glucocorticoid programming of the fetus; adult phenotypes and molecular mechanisms. Mol Cell Endocrinol 2001; 185: 61-71.

57. Reinisch JM, Simon NG, Karow WG, Gandelman R. Prenatal exposure to prednisone in humans and animals retards intrauterine growth. Science 1978; 202: 436-8.

58. Shepherd RW, Stanczyk FZ, Bethea CL, Novy MJ. Fetal and maternal endocrine responses to reduced uteroplacental blood flow. J Clin Endocrinol Metab 1992; 75: 301-7.

\section{Agradecimientos}

Los autores agradecen a los alumnos de pregrado y postgrado de Medicina y de Medicina Veterinaria que colaboraron con los estudios experimentales de ambos laboratorios citados en esta revisión. 